\title{
Should lung ultrasound be always performed in older patients with possible COVID-19 disease?
}

\author{
Costantino Caroselli ${ }^{1} \cdot$ Antonio Cherubini $^{1}$
}

Published online: 13 July 2021

(c) European Geriatric Medicine Society 2021

COVID-19 is the name of the disease caused by the new coronavirus. Old age is a well-known risk factor for developing a symptomatic COVID-19 disease [1,2] and for more severe disease with poor prognosis and high mortality [3, 4]. Available evidence show that COVID-19 mortality sharply increases with age [5-7]. For these reasons, it is extremely important to rapidly identify SARS-CoV2 infection to achieve a point-of-care diagnosis and establish appropriate treatment. Lung ultrasound (LUS) could be invaluable in allowing a quick evaluation of patients with suspected or confirmed COVID-19 disease in different settings, starting from the Emergency Department (ED). First, clinical presentation of SARS-Cov2 infection is different in advanced age compared to the rest of population. In older patients, typical symptoms such as fever or cough [8] might be blunted or even absent [9]. Often atypical symptoms, such as fatigue, anorexia, delirium, falls, and functional decline, might be present $[8,10]$. Therefore, the initial diagnosis is more difficult. Moreover, delirium, which is a well-known presenting condition of COVID-19 infection in older subjects [11], might hinder the diagnostic evaluation and treatment. In older patients with delirium or dementia, even a chest X-ray can be difficult to perform, and even more so, exams such as computed tomography (CT) that require patient collaboration. On the other hand, the use of sedative or antipsychotic medications to perform these diagnostic exams may be counterproductive due to the possibility of adverse effects like, for example, prolongation of the QTc interval or impairment of respiration. On the other hand, a longer stay in the $\mathrm{ED}$ is associated with negative outcomes in older patients, such as delirium or falls [12]. For this reason, rapid and noninvasive diagnostic strategies are important to make an

Costantino Caroselli

costantinocost@yahoo.it

1 U.O.C. Geriatria, Accettazione Geriatrica e Centro di Ricerca per l'invecchiamento, IRCCS INRCA, Ancona, Via della Montagnola, 81, 60127 Ancona, Italy early diagnosis in the ED. Lung ultrasound fulfills all these requirements. It is well established and validated that the Bedside Lung Ultrasound in Emergency (BLUE) protocol [13] plays a pivotal role in the assessment of all patients presenting with acute respiratory failure (ARF) in emergency/ urgency integrating perfectly with the physical examination [14].

Lung ultrasound could be also valuable to support COVID-19 diagnosis and monitoring in out of hospital settings, i.e., at home or in nursing homes, reducing the need to be admitted to the hospital [15-18]. A recent observational research involving 48 older nursing home residents, aged a mean of $84.1 \pm 9.8$ years, undergoing LUS examination made within 3 days from the positivity of nasopharyngeal swab test for COVID-19, demonstrated that LUS plays a significant role in predicting mortality in nursing home residents affected by COVID-19. This study underlines the need to use routinely LUS in this scenario instead of other diagnostic techniques available only in the hospital [16]. There are also practical experiences on how it is possible to carry out lung ultrasound at home in patients affected by SARSCoV2 [19]. In non-hospital settings, lung ultrasound allows to carry out a noninvasive instrumental monitoring by visualizing in real-time any modification of lung patterns and consequently providing precious information about patient clinical conditions, evolution and prognosis, allowing to detect deterioration as well as patient improvement [20].

Concerning the approach to perform LUS in older patients with suspected COVID-19, hereafter practical information is provided, on the basis of the available scientific evidence and experience gained at our center [21]. LUS examination can be performed using high-frequency linear or convex transducers, depending on the need to study better the pleural line or to get a panoramic vision of the lung, as well as operator preference [21]. It is advisable to put the probe along the longitudinal axis using an intercostal approach. This conventional position allows to visualize the upper and lower ribs as reference points and to scan 
the entire corresponding intercostal space and consequently the portion of lung included in the aforementioned intercostal space. Different findings have been reported during LUS in patients with Covid-19 infection: thickening and irregularity of the pleural line; the presence of B-lines with different distribution; focal, multifocal or confluent B-lines evolving in white lung; the presence of focal or multifocal, small or larger consolidations and further classified into translobar or non-translobar. At variance, pleural effusion is an uncommon finding [22]. During the recovery phase a progressive reappearance of A-lines instead B-lines [23] has been reported. The signs and artifacts observed with LUS in COVID-19 patients are similar to those previously reported in patients affected from pneumonia [24] and in patients with acute respiratory distress syndrome (ARDS) [25] and acute lung injury (ALI) [26] presenting a wet lung. The eChoVid study, published as a preliminary report of work on medRxiv, showed that LUS enables identification of lung lesions like chest CT in COVID-19 patients [27].

Concerning the best approach to perform LUS there are discordant views in the literature.

Some authors suggested to study 12 lung areas, 6 areas for each side: anterior upper area and anterior lower area, lateral upper area and lateral lower area, posterior upper area and posterior lower area [23]. At variance, Soldati et al. proposed to evaluate 16 areas ( 8 for each lung) represented from: anterior midclavicular approach (apical, medial and basal), lateral axillary approach (apical, medial and basal) and posterior paraspinal approach (apical, medial and basal) [28]. In different studies, the chest was divided into 6 points (BLUE protocol), 6 zones, 8 zones, 12 zones or 28 zones $[23,24,29]$. To date, there is no universal agreement on which of these protocols is better to use. We retain that all approaches are valid since they are aimed at obtaining a lung aeration score (LUS) useful to measure lung aeration loss caused by different pathological conditions [30, 31]. This is a semiquantitative score represented from the sum of all areas, that allows us to establish the infection severity and to predict the prognosis [32].

However, particularly in an ED setting, a rapid examination is preferred and we suggest to scan 12 lung areas ( 6 for each lung: 2 anterior, 2 lateral and 2 posterior) because the use of protocols with too many zones is too complicated to perform and it is time consuming.

In conclusion, bedside LUS applied to the older patient with suspected interstitial pneumonia secondary to SARSCoV2 infection is coming of age and it should be widely implemented both in hospital and out of hospital settings since it provides relevant information, by defining the extent of lung involvement, the disease evolution and supporting the stratification of the risk of death [33].

\section{Declarations}

Conflict of interest The authors report no conflicts of interest.

\section{References}

1. Zhang J, Wang X, Jia X, Li J, Hu K, Chen G et al (2020) Risk factors for disease severity, unimprovement, and mortality in COVID-19 patients in Wuhan. China Clin Microbiol Infect 26(6):767-772

2. Zhou F, Yu T, Du R, Fan G, Liu Y, Liu Z et al (2020) Clinical course and risk factors for mortality of adult inpatients with COVID-19 in Wuhan, China: a retrospective cohort study. Lancet 395(10229): 1054-1062

3. Gandhi RT, Lynch JB, del Rio C (2020) Mild or moderate COVID-19. N Engl J Med 383:1757-1766. https://doi.org/10. 1056/NEJMcp2009249

4. Ng Cheong Chung KJ (2021) The frailty and mortality relationship in patients with COVID-19. European Geriatric Medicine 12:213-214

5. Onder G, Rezza G, Brusaferro S (2020) Case-fatality rate and characteristics of patients dying in relation to COVID-19 in Italy. JAMA 323(18):1775-1776. https://doi.org/10.1001/jama.2020. 4683

6. Wang L, He W, Yu X, Hu D, Bao M, Liu H et al (2020) Coronavirus disease 2019 in elderly patients: characteristics and prognostic factors based on 4-week follow-up. J Infect. https://doi.org/10. 1016/j.jinf.2020.03.019

7. Perrotta F, Corbi G, Mazzeo G, Boccia M, Aronne L, D’Agnano V et al (2020) COVID-19 and the elderly: insights into pathogenesis and clinical decision-making. Aging Clin Exp Res 16:1-10

8. Guo T, Shen Q, Guo W, He W, Li J, Zhang Y et al (2020) Clinical characteristics of elderly patients with COVID-19 in Hunan Province, China: a Multicenter, retrospective study. Gerontology 66:467-475

9. Norman DC, Yoshikawa TT (1996) Fever in the el-derly. Infect Dis Clin North Am 10(1):93-99

10. Zazzara MB, Penfold RS, Roberts AL, Lee KA, Dooley H, Sudre $\mathrm{CH}$ et al (2020) Probable delirium is a presenting symptom of COVID-19 in frail, older adults: a cohort study of 322 hospitalised and 535 community-based older adults. Age Ageing. https://doi. org/10.1093/ageing/afaa223

11. Blain H, Rolland Y, Benetos A, Giacosa N, Albrand M, Miot S et al (2020) Atypical clinical presentation of COVID-19 infection in residents of a long-term care Facility. Eur Geriatr Med 11:1085-1088. https://doi.org/10.1007/s41999-020-00352-9

12. American College of Emergency Physicians; American Geriatrics Society; Emergency Nurses Association; Society for Academic Emergency Medicine; Geriatric EmergencyDepartment Guidelines Task Force (2014) Geriatric emergency department guidelines. Ann Emerg Med 63(5):e7-25. https://doi.org/10.1016/j. annemergmed.2014.02.008

13. Lichtenstein DA, Mezière GA (2008) Relevance of lung ultrasound in the diagnosis of acute respiratory failure*: the blue protocol. Chest 134:117-125

14. Mongodi S, Orlando A, Arisi E, Tavazzi G, Santangelo E, Caneva L et al (2020) Lung ultrasound in patients with acute respiratory failure reduces conventional imaging and health care provider exposure to COVID-19. Ultrasound Med Biol 46(8):2090-2093

15. Brogi E, Bignami E, Sidoti A, Shawar M, Gargani L, Vetrugno L (2017) Could the use of bedside lung ultrasound reduce the number of chest $\mathrm{x}$-rays in the intensive care unit? Cardiovasc Ultrasound 15(1):23. https://doi.org/10.1186/s12947-017-0113 
16. Veronese N, Sbrogiò LG, Valle R, Marin L, Boscolo E, Tiozzo A (2020) Prognostic Value of Lung Ultrasonography in Older Nursing Home Residents Affected by COVID-19. JAMDA 2020:1384-1386

17. Legramante JM, Morciano L, Lucaroni F, Gilardi F, Caredda E, Pesaresi A et al (2016) Frequent use of emergency departments by the elderly population when continuing care is not well established. PLoS ONE 11(12):e0165939. https://doi.org/10.1371/ journal.pone.0165939

18. Blain H, Rolland Y, Schols JMGA, Cherubini A, Miot S, O'Neill D et al (2020) August 2020 interim EuGMS guidance to prepare european long-term care facilities for COVID-19. European Geriatric Medicine 11:899-913. https://doi.org/10.1007/ s41999-020-00405-z

19. Shokoohi H, Duggan NM, García-de-Casasola Sánchez G, TorresArrese M, Tung-Chen Y (2020) Lung ultrasound monitoring in patients with COVID-19 on home isolation. Am J Emerg Med S0735-6757(20)30440-X

20. Guarracino F, Vetrugno L, Forfori F, Corradi F, Orso D, Bertini $P$ et al (2020) Lung, heart, vascular, and diaphragm ultrasound examination of COVID-19 patients: a comprehensive approach. J Cardiothorac Vasc Anesth. https://doi.org/10.1053/j.jvca.2020. 06.01

21. Norbedo S, Blaivas M, Raffaldi I, Caroselli C (2020) Lung ultrasound point-of-view in pediatric and adult COVID-19 infection. J Ultrasound Med 9999:1-10

22. Peng QY, Wang XT, Zhang LN, Chinese Critical Ultrasound Study Group (CCUSG) (2020) Findings of lung ultrasonography of novel corona virus pneumonia during the 2019-2020 epidemic. Intensive Care Med 12:1-2

23. Lichtenstein DA (2015) BLUE-protocol and FALLS-protocol: two applications of lung ultrasound in the critically ill. Chest 147:1659-1670

24. Volpicelli G, Elbarbary M, Lichtenstein Blaivas M, DA, Mathis G, Kirkpatrick AW, et al (2012) International Liaison Committee on Lung Ultrasound (ILC-LUS) for International Consensus Conference on Lung Ultrasound (ICC-LUS) International evidencebased recommendations for point-of-care lung ultrasound. Intensive Care Med 38:577-591
25. Zhou Y, Fan Q, Cavus O, Zhang X (2018) Lung ultrasound: Predictor of acute respiratory distress syndrome in intensive care unit patients. C Saudi J Anaesth 12(3):457-461

26. Gargani L, Lionetti V, Di Cristofano C, Bevilacqua G, Recchia FA, Picano E (2007) Early detection of acute lung injury uncoupled to hypoxemia in pigs using ultrasound lung comets. Crit Care Med 35:2769-2774

27. Benchoufi M, Bokobza J, Chauvin A, Dion E, Baranne ML, Levan F et al (2020) Lung injury in patients with or suspected COVID19: a comparison between lung ultrasoundand chest CT-scanner severity assessments, an observational study. MedRxiv. https:// doi.org/10.1101/2020.04.24.20069633

28. Soldati G, Smargiassi A, Inchingolo R, Buonsenso D, Perrone T, Briganti DF et al (2020) Is there a role for lung ultrasound during the covid-19 pandemic? J Ultrasound Med 39:1459-1462

29. Buessler A, Chouihed T, Duarte K (2020) Accuracy of several lung ultrasound methods for the diagnosis of acute heart failure in the ED: a multicenter prospective study. Chest 157:99-110

30. Deng Q, Zhang Y, Wang H, Chen L, Yang Z, Peng Z (2020) Semiquantitative lung ultrasound scores in the evaluation and follow-up of critically ill patients with COVID-19: a single-center study. Acad Radiol 27(10):1363-1372

31. Soummer A, Perbet S, Brisson H, Arbelot C, Constantin JM, Lu $Q$ et al (2012) Ultrasound assessment of lung aeration loss during a successful weaning trial predicts postextubation distress. Crit Care Med 40(7):2064-2072

32. Volpicelli G, Lamorte A, Villén T (2020) What's new in lung ultrasound during the COVID-19 pandemic. Intensive Care Med 46:1445-1448

33. Recinella G, Marasco G, Tufoni M, Brizi M, Evangelisti E, Maestri L (2021) Clinical role of lung ultrasound for the diagnosis and prognosis of coronavirus disease pneumonia in elderly patients: a pivotal study. Gerontology 67:78-86

Publisher's Note Springer Nature remains neutral with regard to jurisdictional claims in published maps and institutional affiliations. 